\title{
A NOTE ON THE ESTIMATION OF THE POISSON PARAMETER
}

\author{
S.S. CHITGOPEKAR \\ Department of Management and Marketing \\ Illinois State University \\ Normal, Illinois 61761 U.S.A. \\ (Received August 19, 1983)
}

ABSTRACT. This paper considers the problem of estimating the mean of a Poisson distribution when there are errors in observing the zeros and ones and obtains both the maximum likelihood and moments estimates of the Poisson mean and the error probabilities. It is interesting to note that either method fails to give unique estimates of these parameters unless the error probabilities are functionally related. However, it is equally interesting to observe that the estimate of the poisson mean does not depend on the functional relationship between the error probabilities.

KEY WORDS AND PHRASES. Poisson distribution, estimation, observation errors. 1980 MATHEMATICS SUBJECT CLASSIFICATION CODE. $62 F 10$

\section{INTRODUCTION}

Cohen [1] considers the situation where in observing a Poisson-distributed random variable, some of the values of one are erroneously reported as zero. He obtains the maximum likelihood estimates of the Poisson parameter $\lambda$, and $\theta$, the proportion of ones erroneously reported as zero.

Chitgopekar [2] considers the case where for a Poisson-distributed random variable, while some ones are erroneously reported as zeros, some zeros are also erroneously reported as ones. Chitgopekar [2] observes that unless the error probabilities are functionally related, unique maximum likelihood estimates of the parameters cannot be obtained. Chitgopekar [2] then considers some ad hoc functional relationships between the error probabilities.

In this paper we consider a very general relationship between the error probabilities and observe the interesting fact that the maximum likelihood estimate of the Poisson parameter $\lambda$ is independent of the functional relationship between the error probabilities. 
This paper also considers the estimation of the parameters by the method of moments.

\section{DERIVATION OF THE MAXIMUM LIKELIHOOD ESTIMATES}

Let $X$ be a Poisson random variable with mean $\lambda$. Let $\propto$ be the probability that a value one of $x$ is erroneously reported as zero and $\beta$ be the probability that a value zero of $X$ is erroneously reported as one. The probability function of $X$ in such a situation may be written as:

$$
p(x ; \lambda, \alpha, \beta)=\begin{array}{ll}
(1-\beta) e^{-\lambda}+\alpha \lambda e^{-\lambda} & \text { for } x=0 \\
\beta e^{-\lambda}+(1-\alpha) \lambda e^{-\lambda} & \text { for } x=1 \\
e^{-\lambda \lambda x} / x ! & \text { for } x \geq 2 .
\end{array} .
$$

Since we cannot get unique maximum likelihood estimates of $\lambda, \alpha$, and $\beta$ unless $\alpha$ and $\beta$ are functionally related (Chitgopekar [2]), we assume that

$$
\begin{aligned}
& B=f(\alpha) \\
& \text { where } 0 \leq f(\alpha) \leq 1 \text { for } 0 \leq \alpha \leq 1 .
\end{aligned}
$$

Chitgopekar [2] has considered the particular cases: $f(\alpha)=\alpha, f(\alpha)=\alpha^{2}$, and $f(\alpha)$ $=\sqrt{\alpha}$, whereas Cohen [l] has considered the case $f(\alpha)=0$.

with $\beta=f(\alpha)$, the probability function of $x$ will be

$$
p(x ; \lambda, \propto)=\left\{\begin{array}{ll}
(1-f(\alpha)) e^{-\lambda}+\alpha \lambda e^{-\lambda} & \text { for } x=0 \\
f(\alpha) e^{-\lambda}+(1-\alpha) \lambda e^{-\lambda} & \text { for } x=1 \\
e^{-\lambda} \lambda^{x} / x ! & \text { for } x \geq 2 .
\end{array}\right. \text {. }
$$

Let $x_{1}, x_{2}, \ldots . x_{N}$ be a random sample of size $N$ from this population. Let the number of reported zeros be $n_{0}$ and the number of reported ones be $n_{1}$. Since for a sufficiently large $N, p\left(n_{0}=0\right.$ and $\left.n_{1}=0\right)$ would be very small, we will assume that in our samples $n_{0}>0$ and $n_{1}>0$. The likelihood function for such a sample will be:

$L\left(x_{1}, x_{2}, \ldots . x_{n} ; \lambda, \alpha\right)=$

$$
\left[e^{-\lambda}(1-f(\alpha)+\alpha \lambda)\right]^{n_{0}} \cdot\left[e^{-\lambda}(\lambda-\alpha \lambda+f(\alpha))\right]^{n_{1}} \cdot \operatorname{II}^{*} e^{-\lambda} \lambda^{x} / x !
$$

( II* denotes the product over the values of $x \geq 2$.)

Differentiating $\log L\left(x_{1}, x_{2}, \ldots x_{N} ; \lambda, \propto\right)$ partially with respect to $\lambda$ and $\propto$ and equating to zero, we get

$$
\begin{aligned}
& \frac{T-n_{1}}{\lambda}+\frac{n_{0} \alpha}{1+\alpha \lambda-f(\alpha)}+\frac{n_{1}(1-\alpha)}{\lambda-\alpha \lambda+f(\alpha)}-N=0 \\
& \left(\lambda-f^{\prime}(\alpha)\right)\left[\frac{n_{0}}{1+\alpha \lambda-f(\alpha)}-\frac{n_{1}}{\lambda-\alpha \lambda+f(\alpha)}\right]=0 \\
& \text { where } T=\sum_{i=1}^{N} x_{i} .
\end{aligned}
$$

It can be verified that $\lambda-f^{\prime}(\alpha)=0$ does not provide a maximum of the likelihood function. 
After some algebraic simplification of (2.6), we get

$\alpha \lambda-f(\alpha)=\frac{n_{0} \lambda-n_{1}}{n_{0}+n_{1}}$.

Using (2.7), (2.5) reduces to $\lambda^{2}-\lambda\left(\bar{x}-1+\frac{n_{0}}{N}\right)-\left(\bar{x}-\frac{n_{1}}{N}\right)=0 .(2.8)$ Solution of (2.8) gives the maximum likelihood estimate of $\lambda$. Using this estimate of $\lambda$ in (2.7), we get a maximum likelihood estimate of $\alpha$.

It is interesting to observe that the equation (2.8) is the same as equations (2.8), (3.8), and (4.9) of Chitgopekar [2], and equation (4) of cohen [1]. Further, (2.8) is independent of the function $f$ and thus the maximum likelinood estimate of $\lambda$ is independent of the functional relationship between $\alpha$ and $\beta$.

3. ESTIMATION BY THE METHOD OF MCMENTS

Let $\mu_{k}^{*}$ denote the $k^{\text {th }}$ raw moment of the modified Poisson random variable $x$ whose probability function is given by $(2.1)$ and let $\mu_{k}$ denote the $k^{\text {th }}$ raw moment of the original Poisson random variable with mean $\lambda, k \geq 1$. We then have

$$
\mu_{k}^{*}=(\beta-\alpha \lambda) e^{-\lambda}+\mu_{k}, k \geq 1 \text {. }
$$

Let $m_{k}$ denote the $k^{\text {th }}$ raw moment of the sample, $k \geq 1$. The moments estimators of $\lambda$, $\alpha$, and $\beta$ are obtained by solving

$$
\left.\begin{array}{l}
\mu_{1}^{*}=m_{1} \\
\mu_{2}^{*}=m_{2} \\
\mu_{3}^{*}=m_{3}
\end{array}\right\}
$$

which reduce to

From (3.3) we get

$$
\left.\begin{array}{c}
m_{1}=\lambda+A \\
m_{2}=\lambda^{2}+\lambda+A \\
m_{3}=\lambda\left(\lambda^{2}+3 \lambda+1\right)+A
\end{array}\right\}
$$

Once again, we observe that we cannot estimate the error probabilities separately, and that the estimate of $\lambda$ does not depend on the relationship between the error probabilities.

\section{SIGNIFICANCE OF THE RESULT}

The result that the maximum likelihood estimate as well as the moments estimate of $\lambda$ is dependent of the functional dependence between $\alpha$ and $\beta$ is of great practical significance. In a quality control type setting, one could come across a situation where there are errors in observing a Poisson variable as described in this paper. It would be very unrealistic to expect to know the relationship between the error probabilities. Our results show that for our primary objective of estimating the 
mean of the Poisson distribution, this lack of knowledge of the relationship between the error probabilities is no hurdle.

REFERENCES

1. Cohen, A.C., (Jr). Estimating the Parameters of the Modified Poisson Distribution, Jour. of Amer. Stat. Assn., 1960.

2. Chitgopekar, S.S. Estimation of the Poisson Parameter in the Presence of Errors of Observations. Proceedings of the 13th Annual Meeting of AIDS, Vol. 2, 1981. 


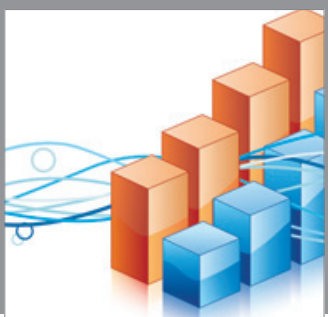

Advances in

Operations Research

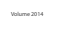

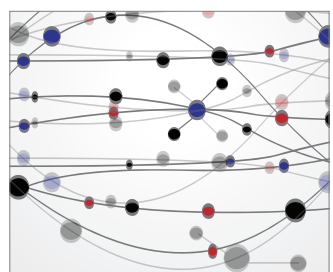

\section{The Scientific} World Journal
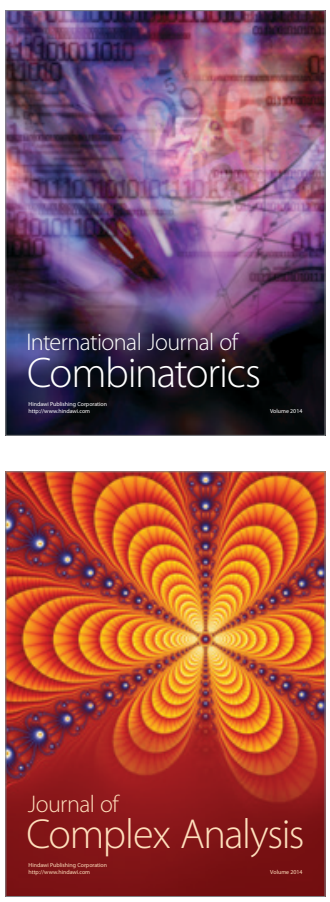

International Journal of

Mathematics and

Mathematical

Sciences
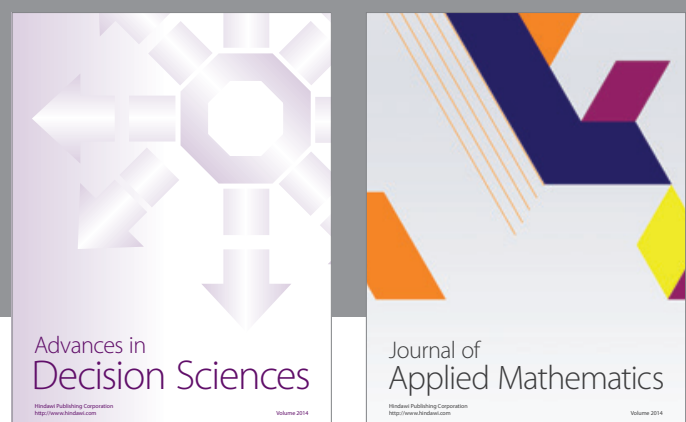

Journal of

Applied Mathematics
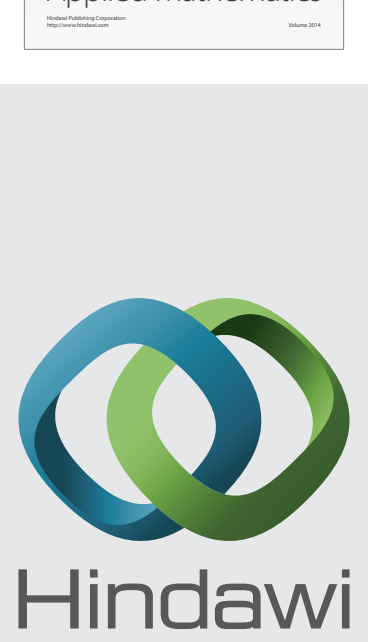

Submit your manuscripts at http://www.hindawi.com
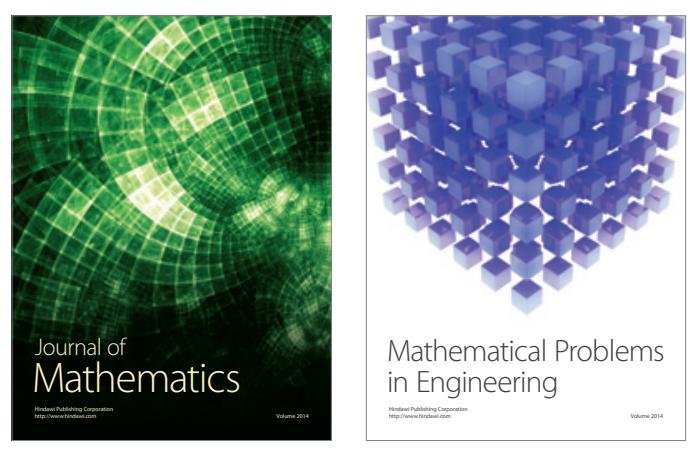

Mathematical Problems in Engineering
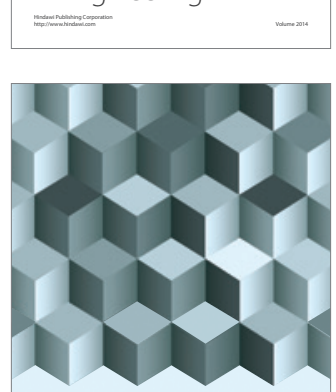

Journal of

Function Spaces
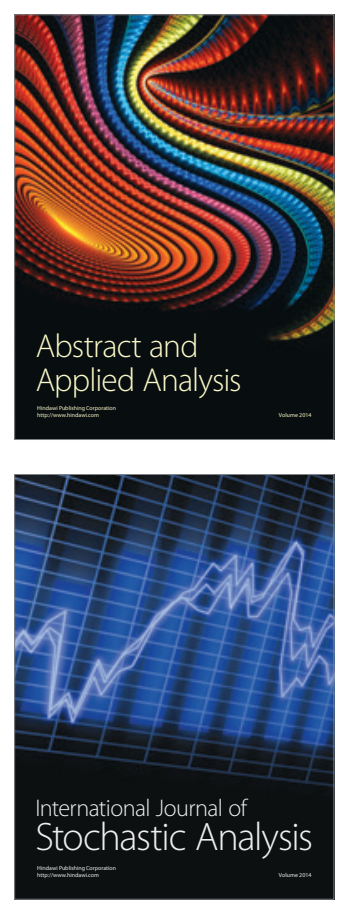

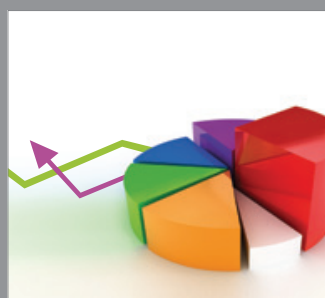

ournal of

Probability and Statistics

Promensencen
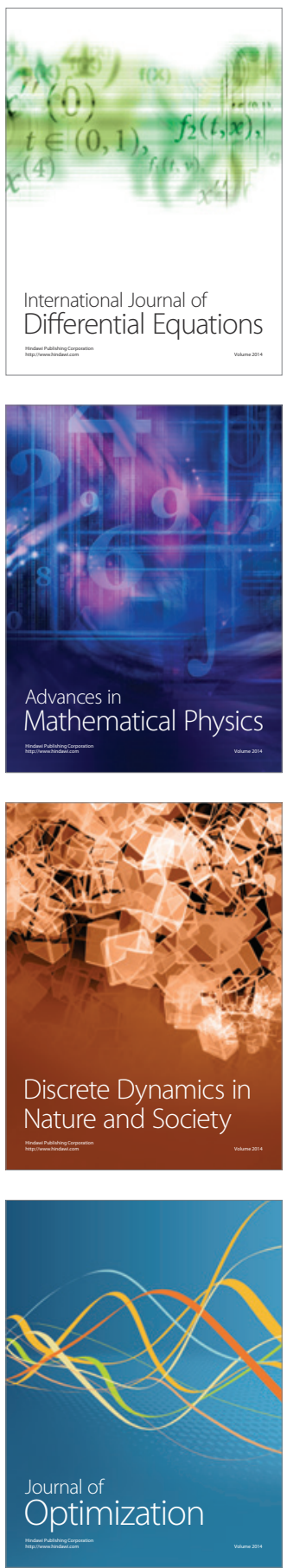